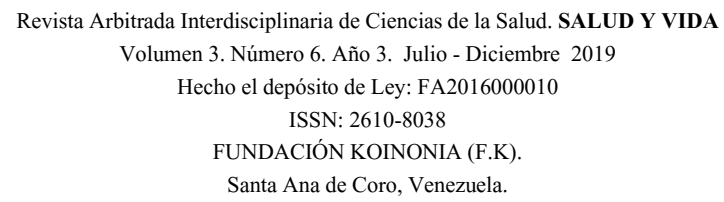

Julio Juvenal Aldana Zavala; Josía Jeseff Isea Argüelles

http://dx.doi.org/10.35381/s.v.v3i5.363

\title{
La economía y la epidemiología social. Una praxis humana en la cosmovisión del nuevo milenio
}

The economy and social epidemiology. A human praxis in the worldview of the new millennium

\author{
Julio Juvenal Aldana Zavala \\ julioaldanazavala@gmail.com \\ Universidad Nacional Experimental Francisco de Miranda \\ Venezuela \\ https://orcid.org/0000-0002-7934-9103 \\ Josía Jeseff Isea Argüelles \\ josiaisea@gmail.com \\ Universidad Nacional Experimental Francisco de Miranda \\ Venezuela \\ https://orcid.org/0000-0001-8921-6446
}

Recibido: 27 de octubre del 2018

Aprobado: 30 de noviembre del 2018

\begin{abstract}
RESUMEN
El artículo tiene como propósito analizar la economía y la epidemiología social como una praxis humana en la cosmovisión del nuevo milenio, esto implica plantearse un modelo social que permita procesar la salud como un ente de prevención desde los multifactores que conforman la sociedad, es decir, economía, educación, política, son una triada que influyen positiva o negativamente en la incidencia de la salud pública, siendo pertinente promover un enfoque que permita a la población desarrollar una cultura para la vida.
\end{abstract}

Descriptores: Política de salud; Epidemiología; Medicina Preventiva; Sistema económico. 


\begin{abstract}
The article aims to analyze the economy and social epidemiology as a human praxis in the worldview of the new millennium, this implies considering a social model that allows to process health as a prevention entity from the multifactors that make up society, that is, Economy, education, politics, are a triad that positively or negatively influence the incidence of public health, being relevant to promote an approach that allows the population to develop a culture for life.
\end{abstract}

Descriptors: Health policy, epidemiology, preventive medicine, economic systems.

\title{
A modo introductorio
}

La humanidad se ha enfrentado a dos milenios y se encuentra en desarrollo de un tercero. En el primero, se desplegó la filosofía antigua en el mundo occidental, desde el auge de Grecia hasta la caída del Imperio Romano, caracterizando en primer orden por las investigaciones naturalistas y en segundo, con la aparición de Sócrates, el estudio del ser humano en su vida en la sociedad. Desarrollándose así, un giro antropológico en la esencia del estudio efectuado por los filósofos de la época. Por otro lado, el segundo milenio dista desde el 1 de enero del 1001 al 31 de diciembre del 2000.

El segundo milenio, inicia con el fin del mundo antiguo occidental y se desarrolla la filosofía medieval como epicentro de estudio. La fe cristiana fue el centro de investigación, las concepciones Platónicas y luego Aristotélicas, sirvieron de base para el desarrollo entre la fe y la razón, aunque se efectuó un punto de quiebre donde la fe se impuso sobre el estudio de la razón, siendo así, que todo cuestionamiento debía ser sustentado desde las Sagradas Escrituras, siendo la Iglesia Católica, el ente rector encargado de aprobar o desaprobar el pensamiento científico. Por ende el Renacimiento representó un punto de quiebre en la cosmovisión de estudiar y entender el mundo; el humanismo como corriente de pensamiento surgió como repuesta a la fe como único ente de acceso a la "verdad", siendo que el humanismo la revalorización del ser humano como centro de estudio. 
Este nuevo sentir de la búsqueda de la verdad, dio origen a la filosofía moderna, René Descartes desde el método (duda) como vía de acceso al conocimiento científico, le brindó a la "razón" el valor necesario para promover nuevamente la discusión con base al "logos", alejándose progresivamente de los preceptos de validación cristiana como fuente exclusiva del conocimiento. Otros pensadores como Immanuel Kant y Georg Wilhelm Friedrich Hegel, complementaron desde sus perspectivas el estudio del Hombre como centro de investigación, el primero desde el ideal de transcendencia y el segundo con la dialéctica, establecieron sistemas que permitieron la confrontación filosófica sobre el devenir del Hombre y su desarrollo en el mundo, de ese modo, se desarrolló la filosofía moderna.

Por consiguiente, apareció la filosofía contemporánea (muerte de Hegel 1831 hasta nuestros días) en donde exponentes como Friedrich Nietzsche y Martín Heidegger plantean que el Hombre se revela como aquel que juzga, aquel que da sentido. Por lo tanto, el mundo tiene el sentido y el valor que el ser humano le brinda, siendo esta concepción quien brinda transcendencia al pensamiento humano como fuente hacia el conocimiento científico. Podría señalarse que la etapa antigua de la filosofía (I Milenio) se centró en estudiar al "Mundo". El segundo Milenio dividido en filosofía Medieval (estudió a Dios), Moderna (estudió al Hombre), y Contemporánea (Se destacó el cuestionamiento sobre "la imposibilidad de un saber absoluto").

Siguiendo con lo planteado, en la filosofía antigua los investigadores tuvieron una actitud de "asombro" ante el mundo que les rodeaba. En lo medieval fue una actitud de "Fe", en la moderna de "duda" y en la contemporánea de "ambigüedad". Ahora bien, en este naciente Tercer Milenio surge la interrogante ¿Cuál es la actitud del filósofo o del investigador ante la realidad? Una pregunta difícil de responder a priori, más aun, cuando en el presente Milenio se habla de la etapa de la filosofía postmoderna o postmodernismo; el cual para muchos pensadores no se tiene una clara definición del mismo y se prefiere señalar que el mundo se encuentra en una "transición" a la postmodernidad. 


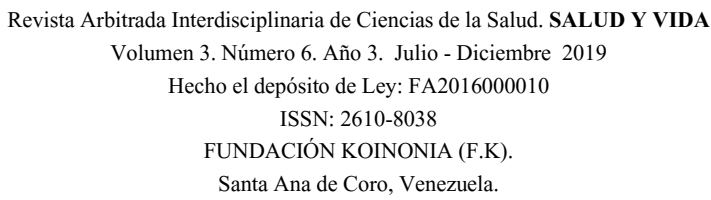

Es de destacar, que el debate del actual manuscrito no radica en dilucidar tal incertidumbre, sino generar una alternativa sobre la cosmovisión del investigador en el campo de la "economía y la epidemiología social", como generadores de una praxis humana en concordancia en transcender prácticas mecanicistas donde el ser humano es percibido como un objeto, como una mercancía, distante de los nuevos enfoques del pensamiento, entre el cual se encuentra el sistémico donde se estudian las partes como un todo complejo, de ese modo, la cosmovisión transciende a entender que el mundo es global y que el ser humano habita en un ecosistema el cual debe mantener, preservar, para las futuras generaciones. En tal sentido, Deming (1994: 12) señala que "El pensamiento lineal es lo opuesto al pensamiento sistémico. Pensamos linealmente cuando buscamos culpables o cuando, en general, buscamos la causa inmediata de algún evento". Por consiguiente, el Tercer Milenio debe destacarse por acciones complejas, complementarias, sistémicas, coadyuvantes en solucionar los problemas que afectan la humanidad, siendo uno de ellos; la economía y el otro la epidemiología.

La economía porque en ella se desarrollan las relaciones de poder entre los seres humanos, en las sociedades ocurre con frecuencia que quienes tienen la mayor suma de dinero pueden controlar al resto de la población. Los ciudadanos en la medida que tienen poder adquisitivo son clasificados en estratos sociales, determinando así, el valor social de ese segmento poblacional. El dinero puede ser utilizado de diversos modos, bien sea para invertir en bienes o servicios que a su vez produzcan mayores bienes (capital privado) o de inversión social para el desarrollo de la nación (Estado).

Luego el campo de la salud juega un papel fundamental en el desarrollo integral del ser humano, bien sea desde una perspectiva privada donde la persona según sea su capacidad económica puede aspirar al sufragio de los gastos necesarios para cubrir sus necesidades médicas o bien sea por el lado del Estado, donde por medio de políticas públicas contribuyan al acceso de las personas a la salud. Estos modelos económicos del capital privado o público generan prácticas en el ser humano desde una perspectiva consumidora, bien sea del dinero o de la salud. En esta última no se maneja el modelo 
de "prevención integral" que fusiona lo económico con lo epidemiológico para establecer principios de complejidad y complementariedad de los fenómenos sociales, ante lo cual, es necesario transcender la cultura donde lo económico, político, sanitario, van por vertientes diferentes cuando en realidad son ente holístico integrado desde las partes del desarrollo social del ser humano.

Desde lo planteado, parece ahora tener mayor proyección en encontrarse la repuesta a la interrogante ¿Cuál es la actitud del filósofo o del investigador ante la realidad en este tercer milenio? Seguramente no debe ser la de estudiar la realidad desde una visión fragmentada - mecanicista - lineal. Quizás sea más cónsona la de pensar en una perspectiva integral - holística - complementaria - sistémica - compleja de estructurar el conocimiento, por cuanto esto garantiza generar una praxis basada en el dinamismo humano y su relación con su cultura, la cual marca un desarrollo con los otros, es decir con el Alter Ego. En consecuencia, sería un accidente pensar que lo económico y epidemiológico transitan caminos paralelos, por el contrario en el nuevo milenio han de transitar simbióticamente para estructurar salidas a las múltiples problemáticas que atraviesa la humanidad, por solo mencionar el tema del agua y de la agroalimentación ¿Acaso estas no inciden en lo económico y en lo epidemiológico?

Desde la perspectiva del autor, el Tercer Milenio debe estar marcado por una actitud hacia la transcendencia en la resolución de los conflictos del ser humano en concordancia con mantener el equilibrio del ecosistema donde habita. Esta ingenuidad filosófica permite avizorar un mundo más justo donde el conocimiento científico contribuya al equilibrio mundial, seguir actuando bajo la cultura que ha conllevado al mundo al foso donde se encuentra hoy, es simplemente esperar la destrucción total del mismo.

\section{La economía social ante el naufragio mundial}

Cada milenio anterior ha traído consigo una ruptura de viejas prácticas, nuevos modos de acceso al conocimiento, entre otros elementos que han reconfigurado las praxis humanas en aras de estructurar acciones coadyuvantes en el desarrollo integral de la 
humanidad. Cada momento histórico les brinda a sus actores, pinceladas de certeza así como de incertidumbre, hoy por hoy el mundo se encuentra en esta dicotomía. Si seguir anclado a las tradicionales prácticas economicistas o experimentar modelos que no sólo desarrollen lo económico, sino lo humano.

A este último, podría denominarse "economía social", enfoque económico que se presenta como un salvavidas ante el naufragio donde está la humanidad en el campo de lo económico, debido que las estructuras capitalistas fundadas en la seguridad que brindaban sus instituciones así lo develan, basta con solo mirar la crisis de 2008 que sufrió Estados Unidos para recordar que estamos en un mundo donde no existen certezas duraderas, sino, por el contrario incertidumbres hacia dónde ir y que hacer, no solo desde el punto de vista de la economía, sino del conocimiento, de lo social.

La economía social tiene sus bases fundacionales en los postulados cooperativos generados en el siglo pasado, constituyéndose en eslabón con el actual milenio, sin embargo, esta visión aún no está consolidada como praxis económica, debido al arropamiento del sistema capitalista generadora de una conducta consumista del ser humano, siendo la económica social, una tendencia de empresa mixta, es decir, desarrollada entre lo público y lo privado. Esto no implica la inexistencia del capital o de producción de bienes, servicios, dinero, solo que este al ser generado bajo otra perspectiva de trabajo, no solo sirve de beneficios a los empresarios productores, sino, al colectivo social. En este sentido, se comparte la visión de Celis (2003: 22) sobre la definición de cooperativas en donde esta se proyecta como una empresa bajo los preceptos de la economía social.

La cooperativa viene a representar un medio de producción donde la participación democrática y protagónica de los ciudadanos, es un elemento clave para el desarrollo socio económico de la propia organización, así como de la sociedad. En concordancia, se comparte visión epistémica con Batistas y Richer (2001: 5) sobre el hecho de que la economía social se establece como un bloque económico que navega en medio de océanos gestores de prácticas culturales determinantes en la conducta del colectivo. 
Para transcender es necesario gestar en los ciudadanos una óptica de pensamiento basada en lo introspectivo vivencial del sujeto - sujeto como medio de relaciones para el forjamiento de una estructura social horizontal donde los pares ejerzan mediante el diálogo, la empatía, deberes y derechos por parte de las personas para proyectar desde lo económico social, una sociedad con fines administrativos desde la gerencia circular. Es antagónico pensar que la economía social podrá brindar frutos, sí las personas que conforman las estructuras empresariales, trabajan y se comportan con una visión capitalista desde la perspectiva de pensamiento sujeto - objeto, por cuanto las prácticas determinarán a condenar el fracaso a las empresas de la economía social, basta con mirar en retrospectiva los avances jurídicos, de inversión, realizadas a las cooperativas en Venezuela durante el Gobierno de Hugo Chávez Frías, con la finalidad de constituirlas en empresas alternativas al mercado capitalista, sin embargo, en su gran mayoría no brindaron la productividad esperada, por cuanto se instalaron cooperativas, pero quienes las manejaron, lo hicieron con una visión totalmente distinta a los patrones del deber ser de una cooperativa. Esta concepción se fundamenta en lo expresado por la Cooperativa Gestión Participativa (2016: 1).

Esto proyecta que no solo es necesario invertir dinero y generar las condiciones jurídicas para el establecimiento de la economía social, es menester crear una cultura donde la persona transite en lo introspectivo vivencial como modelo de vida para así, poder tener la visión humana de visualizar al otro como una persona de carne y hueso con vida, pensamiento, propio, dinámico, flexible, autónomo, en capacidad de ejercer un liderazgo transformador de las realidades comunitarias en donde se desenvuelve.

La gerencia circular se constituye en una herramienta primordial para el ejercicio de la economía social, es impensable desarrollarla bajo principios gerenciales jerárquicos o piramidales propios de la visión capitalista. Esto es antagónico al hecho mismo de llamarse economía social. Sin el ejercicio circular de la gerencia, es enviar un mensaje en medio del naufragio en el cual vive el mundo actual, el cual no llegará a su destino, debido que será devorado por las enormes olas de la cultura económica tradicional. Sin 
embargo, esta última se encuentra en una profunda crisis pero sus inventores protectores preferirán morir con ella, antes de pensar actuar bajo principios de la economía social.

Las instituciones y gobiernos emergentes tienen el deber moral de proyectarse hacia la consolidación del modelo de economía social como principio activo de sus sociedades. De ese modo, bajo una relación ganar - ganar podrán emerger culturas que internalicen el pensamiento introspectivo vivencial como medio de generación de conductas sociales coadyuvantes en personificar el deber ser de los postulados de la economía social. Desde lo planteado, se establece que el problema central no se encuentra sí se produce capital o no, de hecho este es necesario para poder establecer relaciones comerciales solidas con otras personas, empresas, instituciones, gobiernos, etc. De lo que se trata en cuestión es de cómo se comportan y actúan las personas generadores de este capital frente a la realidad social donde se desenvuelven.

Desde la economía social se está llamado a contribuir en la transformación del mundo y no a su destrucción. Por destrucción podemos entender el populismo como fuente de financiamiento de los males de la sociedad, por el contrario, el rol transformador significa dignificar al ser humano por medio de una educación liberadora por medio de un aprendizaje a lo largo de la vida. Las instituciones educativas no solo deben verse como entidades bancarias del conocimiento, sino, como entes holísticos formadores de emprendedores que transciendan la cultura del "asalariado" propia del sistema capitalista tradicional.

Por ende la economía social debe encontrar adeptos que desde la escuela e instituciones de salud contribuyan a fomentar un pensamiento vivencial - holístico para entender la realidad como un sistema complejo en donde se unan esfuerzos en promover estrategias empresariales que contribuyan a consolidar lo educativo y lo epidemiológico como factores primordiales de una economía social en favor de las transformaciones sociales del mundo actual. 


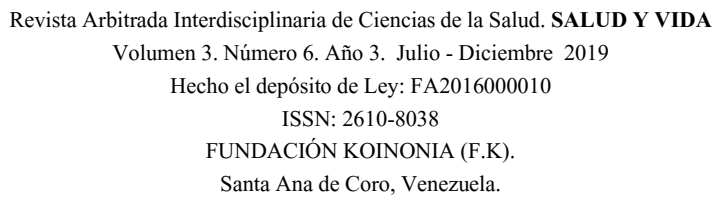

Julio Juvenal Aldana Zavala; Josía Jeseff Isea Argüelles

Es necesario establecer políticas públicas que favorezcan no solo el financiamiento económico, sino el formativo - preventivo para poder contar con actores sociales en capacidad de asumir el rol protagónico y democrático de un líder económico social al servicio de la sociedad y no al contrario. Es allí en donde se encuentra la incertidumbre, si seguir anclados a lo tradicional o darle paso a una estructura económica social con pertinencia en la inversión para el desarrollo integral de la sociedad.

\section{Lo epidemiólogo al servicio de la economía social}

La vertiente de la epidemiologia social, la cual busca estudiar desde las relaciones interpersonales y su incidencia en la salud, se busca transcender el modelo curativo de la salud, tratándose más bien de una perspectiva preventiva e integral de las enfermedades que aquejan al ser humano. La economía social encuentra un aliado para desarrollar un modelo de vida que transcienda lo lineal - mecanicista hasta ahora empleado, el cual ha evidenciado como la persona se enferma y asiste al médico en procura de una cura. Desde esta linealidad, no se abordan los diversos factores que afectan a la humanidad y que podrían subyugar en evitar enfermedades que contrapongan el bienestar individual - colectivo de las personas.

La economía social como empresa al servicio de la sociedad en conjunto con la epidemiologia social, se constituyen en un nuevo modelo de acción donde la empresa puede invertir dinero no solo para la prevención de enfermedades, sino, para la formación de un ciudadano en capacidad de entender que su cuerpo es una maquina al servicio de la sociedad y por ende, debe brindársele la atención preventiva a fin de poder tener mayores condiciones para disfrutar de la vida. Así mismo, se proyecta una visión holística - ecológica del medio donde habita el ser humano, es decir, debe comprenderse que todo lo que actúa en el ambiente puede ser beneficioso o perjudicial para la salud.

Esta interrelación proyecta empresas que sean manejadas por personas con visión integral - vivencial del ecosistema donde habitan, generando una cultura ecológica donde el ser humano es el principal protagonista de las acciones que se desenvuelve allí. Es 
pertinente desde la visión sistémica del pensamiento, generar políticas públicas contribuyentes en promover acciones concatenantes de un accionar humano complejo en donde se visualicen por medio de las interacciones sociales, un devenir histórico social coadyuvante a brindar a la humanidad, alternativas onto epistémica del accionar económico - epidemiológico hacia lo social en procura de transformar la sociedad, para esto es necesario crear nuevos imaginarios sociales que transciendan lo culturalmente establecido, generando desde el interaccionismo simbólico, nuevos significantes de la propia construcción de los actores sociales.

En concordancia con lo planteado, puede considerarse que la fusión de la economía y epidemiologia social, conformaran un bloque para la generación de constructos sociales dinámicos en donde se geste el camino para consolidar una alternativa para la vida y durante la vida, en conformidad con las nuevas tendencias epistémicas hacia donde se moviliza el ser humano del Tercer Milenio.

\section{La economía y epidemiología social. Un sistema alternativo a los 17 objetivos de desarrollo sustentable}

En el Tercer Milenio la humanidad se encuentra hacia el gran reto de asumir en conjunto la resolución de 17 objetivos sustentables que permitirán concretar mayor calidad de vida de cara al 2030. Los primeros 30 años del milenio enfrentan el desafío de superar las diferencias sociales existentes actualmente. Desde esta visión la economía y epidemiología social se constituyen en un sistema alterno para lograr tal fin, por cuanto los objetivos sustentables están determinados en "Poner fin a la pobreza en todas sus formas en todo el mundo".

Al observarse cada uno de los objetivos planteados por la ONU, existe una conexión entre los mismos para el desarrollo integral de la humanidad, pero además, se puede reseñar la disposición que tiene cada uno de brindarle participación protagónica a la economía y epidemiología social como entes de transformación por medio del cual, se puede generar una nueva cultura humanizante, donde lo científico, tecnológico, 


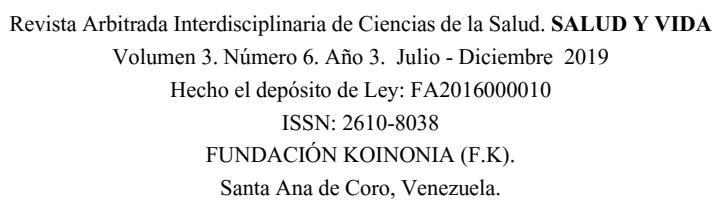

Julio Juvenal Aldana Zavala; Josía Jeseff Isea Argüelles

educativo, coadyuven a estas ramas de la economía y de la salud, a aunar esfuerzos en pro de establecer acciones complejas, sistemáticas, visionarias de una nueva civilización que debe tener en consideración desde lo ecológico - bio ético - como ejes medulares para la construcción de una sociedad basada en proteger a su mejor y mayor especie planetaria como lo es la raza humana.

Es menester vincular el sistema educativo en todos sus niveles y modalidades hacia una transformación curricular, donde se trabaje desde el pensamiento introspectivo vivencial, en una relación epistémica sujeto - sujeto, mediante un diálogo constructivo de saberes como sustento ontoepistémico hacia un accionar pedagógico holístico, complejo, sistémico, de los procesos formulados en las instituciones educativas, bajo una concepción de la epidemiología y economía social, como aristas innovadoras en premisa de integrar en nodos críticos, proyectos de aprendizaje donde se trabaje multi e interdisciplinarmente en la consolidación de un proceso formativo de una nueva ciudadanía en conciencia de cara a los nuevos retos en el devenir global de las próximas décadas.

En síntesis, se resalta la economía social, epidemiología social, educación como elementos, integradores desde donde se debe trabajar para la formación - promoción de un nuevo estilo de vida que permita al ser humano transcender hacia la visualización actuación en el mundo bajo una cosmovisión holística - ecológica de los procesos sociales en los cuales se desenvuelven, ante los desafíos del cambio climático, inestabilidad económica de las grandes potencias, acciones que desencadenan consecuencias en los países menos desarrollados o progresistas, la trilogía de la economía social, epidemiología social, educación, subyacen como una alternativa ante la cual, se puede conformar un sistema de vida con base a la transformación social bajo principios sustentables como ecosistema de vida al servicio de la preservación de las especies que hacen vida en el globo terráqueo, en especial al humano. 


\section{REFERENCIAS CONSULTADAS}

1. Batistas y Richer (2001). Economía social y economía solidaria: Intento de definición. CAYAPA Revista Venezolana de Economía Social. Año 1. № 1. Mayo 2001

2. Cooperativa Gestión Participativa (2016). Explosión cooperativa en Venezuela. ¿Éxito 0 fracaso? [Documento en línea]. Disponible: http://www.gestionparticipativa.coop/portal/index.php?option=com_content\&view $=$ article\&id=173: coopescuela\&catid=3:newsflash\&ltemid=204 [Consulta: 2016, Noviembre 08].

3. Celis, A. (2003). El Nuevo Cooperativismo. Caracas. Vadell Hermanos Editores.

4. Deming, W. E. (1994). La nueva economía: para la industria, el gobierno y la educación. Díaz de Santos, Madrid, España.

5. ONU (2015). Objetivos de desarrollo sustentable. 17 objetivos para la transformación de nuestro mundo. [Documento en línea]. Disponible: http://www.un.org/sustainabledevelopment/es/objetivos-de-desarrollo-sostenible/ [Consulta: 2016, Noviembre 09].

\section{REFERENCES CONSULTED}

1. Batistas and Richer (2001). Social economy and solidarity economy: Attempted definition. CAYAPA Venezuelan Social Economy Magazine. Year 1. No. 1. May 2001

2. Participatory Management Cooperative (2016). Cooperative explosion in Venezuela. Success or failure? [Online document]. Available:

http://www.gestionparticipativa.coop/portal/index.php?option=com_content\&view $=$ article\&id=173: coopescuela\&catid=3:newsflash\&ltemid=204 [Consultation: 2016, November 08].

3. Celis, A. (2003). The New Cooperativism. Caracas. Vadell Brothers Editors.

4. Deming, W. E. (1994). The new economy: for industry, government and education. Díaz de Santos, Madrid, Spain. 


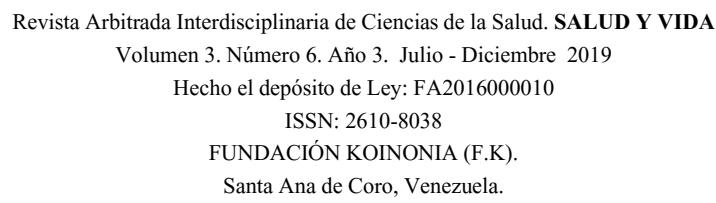

Julio Juvenal Aldana Zavala; Josía Jeseff Isea Argüelles

5. UN (2015). Sustainable Development Goals 17 objectives for the transformation of our world. [Online document]. Available:

http://www.un.org/sustainabledevelopment/es/objetivos-de-desarrollo-sostenible/ [Consultation: 2016, November 09].

2019 por los autores. Este artículo es de acceso abierto y distribuido según los términos y condiciones de la licencia Creative Commons Atribución-NoComercial-Compartirlgual 4.0 Internacional (CC BY-NCSA 4.0) (https://creativecommons.org/licenses/by-nc-sa/4.0/). 